\title{
Síndrome biopercular producido por lesión unilateral
}

\author{
MARGARITA-MARÍA GIRALDO-CHICA ${ }^{1}$, JUAN-DIEGO RAMÍREZ², \\ CLARAMONIKA URIBE ${ }^{1, a}$, FRANCISCO LOPERA ${ }^{1}$
}

\section{Biopercular syndrome caused by unilateral ischemia. Report of one case}

Biopercular syndrome is a labio-facio-pharyngeal-laryngeal-gloso-masticatory diplegia, with automatic dissociation of movements. Ischemia is the most common etiology when it occurs bilaterally in the opercular area, but it has been also described in patients with bilateral subcortical lesions. There are few cases described with unilateral lesions. We report a 76-year-old woman who developed a biopercular syndrome caused by unilateral ischemic lesion of the right middle cerebral artery confirmed by magnetic resonance imaging and cerebral SPECT.

(Rev Med Chile 2010; 138: 341-345).

Key words: Diplegia, spastic; Ischemia; Middle cerebral artery.

\author{
${ }^{1}$ Grupo de Neurociencias \\ de Antioquia. \\ ${ }^{2}$ Grupo de Neurociencia \\ de Caldas. \\ ${ }^{a}$ Fonoaudióloga \\ Apoyo Financiero: Deseamos \\ agradecer a COLCIENCIAS y \\ la Universidad de Antioquia \\ por el financiamiento del \\ proyecto: 1115-343-19127 y \\ al CODI por el financiamiento \\ del programa de Sostenibili- \\ dad 2009-2010 del Grupo de \\ Neurociencias de Antioquia. \\ Las fuentes de financiación \\ influyeron sobre la presenta- \\ ción de la información. \\ Recibido el 10 de junio de \\ 2009, aceptado el 17 de \\ diciembre de 2009. \\ Correspondencia: \\ Margarita-María Giraldo-Chica. \\ Fax: (57)(4) 2196444. \\ E-mail: mgiraldoch@une.net.co
}

$\mathrm{E}$ 1 síndrome de Foix-Chavany-Marie (FCM) o síndrome biopercular (SB) fue descrito como una diplejía labio-facio-faringolaringo-gloso-masticatoria, con disociación automática del movimiento. Los pacientes que padecen este síndrome presentan alteración del habla (disartria o anartria), disfagia y no pueden abrir la boca, cerrar los párpados o protruir la lengua por voluntad propia, pero pueden sonreír, llorar y bostezar automáticamente. La etiología más frecuente en la edad adulta es la isquémica, a nivel opercular bilateral. Presentamos un caso clínico de una paciente con enfermedad cerebrovascular isquémica de la arteria cerebral media derecha con parálisis pseudobulbar. Lo más llamativo de este caso clínico es que una lesión unilateral del opérculo, demostrada por resonancia magnética (RM) de encéfalo y tomografía estandarizada de emisión de fotón único (SPECT) cerebral, produzca compromiso bilateral de los pares craneanos $\mathrm{V}$, VII, IX, X y XII.

\section{Caso clínico}

Mujer de 76 años, diestra, con antecedentes de hipertensión arterial, dislipidemia e hipotiroi- dismo, quien sufrió enfermedad cerebrovascular (ECV) isquémica de la arteria cerebral media derecha. En la evaluación neurológica realizada a los 12 meses del evento, se observó facies inexpresiva, babeo, anartria, sin alteraciones en la esfera mental, obedecía órdenes sencillas, expresaba sus deseos por escrito, sin déficit motor de las extremidades, con dificultades para la deglución, reflejo nauseoso disminuido, sin ascenso del velo del paladar, ni protrusión de la lengua y anartria con actos reflejos (sonrisa, llanto y bostezo) espontáneos pero no por orden verbal. Los reflejos osteotendinosos estaban exaltados en el lado izquierdo con respuesta plantar flexora bilateral.

Los exámenes de sangre realizados al ingreso (hemoleucograma, glicemia, VDRL, TSH, BUN, creatinina, ionograma, pruebas de coagulación) fueron normales. La ecografía dúplex carotídea y vertebral mostró ateromatosis sin estenosis significativa, la ecocardiografía evidenció insuficiencia trivial aórtica y tricuspídea con fracción de eyección de $75 \%$. El Holter mostró ritmo sinusal con importante bradicardia.

En la tomografía computada (TC) de cráneo (Figura 1) se observó un infarto en el territorio irrigado por la arteria cerebral media derecha. 


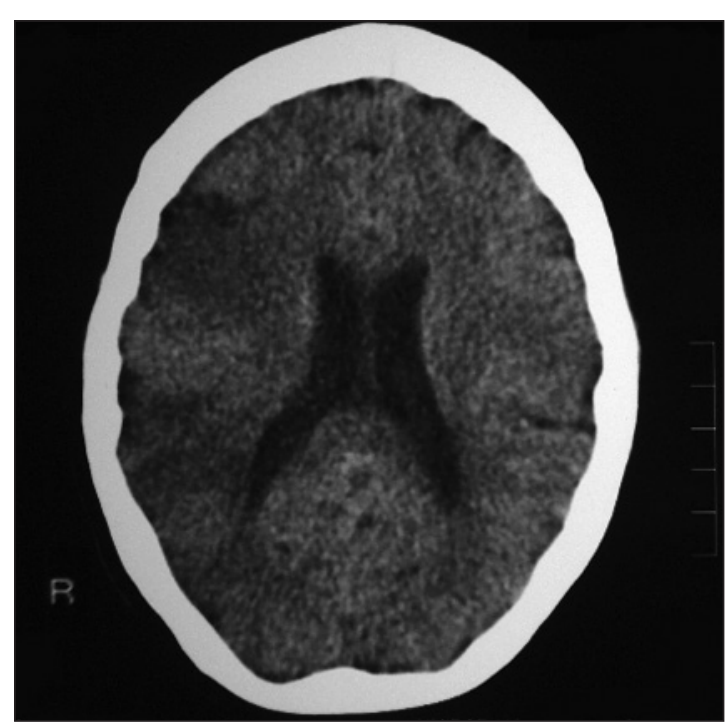

Figura 1. Imagen de TC simple de cráneo que muestra hipodensidad en el área de la arteria cerebral media derecha, el día del ingreso de la paciente.

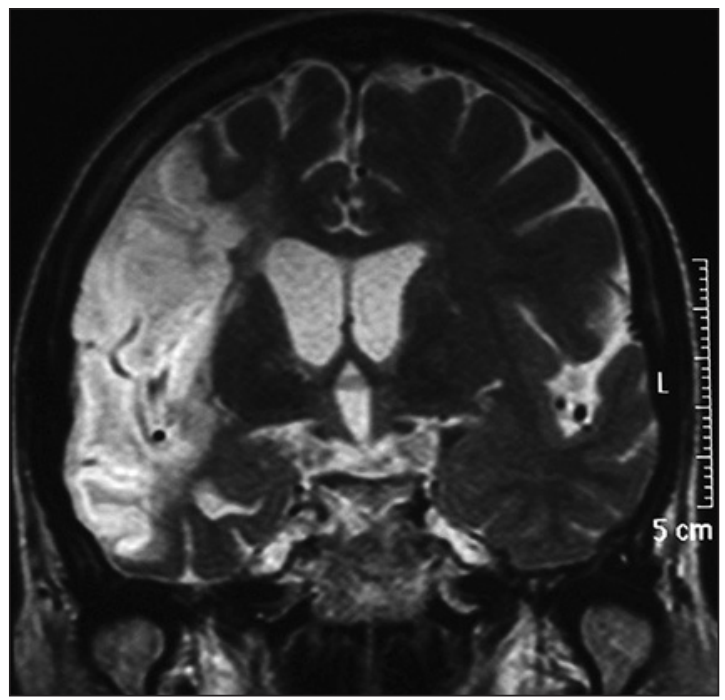

Figura 2. Resonancia magnética de encéfalo, secuencia de T2 en el plano coronal 18 meses después del infarto cerebral, donde se observa hiperintensidad de los lóbulos temporal y parietal derechos, con dilatación del sistema ventricular.

Figura 3. SPECT cerebral realizado con la técnica ${ }^{99 m} \mathrm{TC}-\mathrm{HMPAO}$ que muestra gran zona de hipoperfusión sobre el opérculo frontal, temporal y parietal, sólo en el lado derecho, sin compromiso aparente del lado izquierdo.

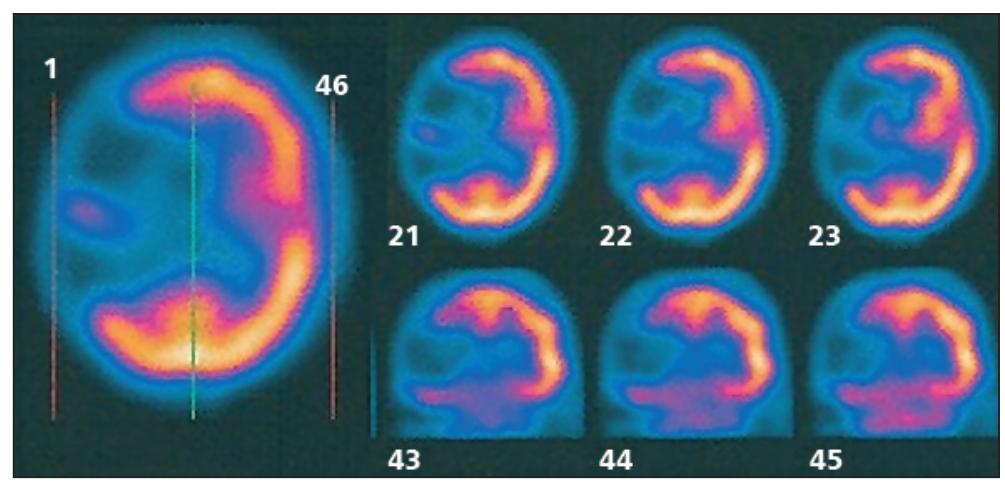

En la resonancia magnética del encéfalo (Figura 2), realizada a los 18 meses del evento, se observó encefalomalacia y gliosis en territorio de la arteria cerebral media derecha después de la unión m2-m3, con dilatación del sistema ventricular en forma compensatoria, sin hallazgos sugerentes de lesión en la región izquierda. En la tomografía estandarizada por emisión de fotón único (con la técnica ${ }^{99 m}$ TC-HMPAO) (Figura 3) se mostró un área hipoperfusional importante que comprometía la región frontal media, posterior y región parietal únicamente del hemisferio derecho.
En la evaluación neuropsicológica se encontró una paciente anártrica, con labilidad afectiva, colaboradora, con lentitud psicomotora, se comunicaba por escrito, lograba expresar sus inquietudes y deseos, orientada en tiempo, espacio y persona, sin alteración en la comprensión, con agnosia espacial unilateral izquierda la cual se evidenció cuando comenzó a escribir en la parte derecha de la hoja y al copiar el reloj sólo escribió los números de la derecha. A los 18 meses, había mejoría la lentitud psicomotora, el estado anímico y la agnosia espacial. 
En la evaluación por fonoaudiología de las características motoras de su habla a través de la observación directa se encontró: Buena comprensión del lenguaje a nivel conversacional con comunicación adecuada mediante la escritura. Anartria: ausencia de lenguaje expresivo oral por Incapacidad motora para realizar de manera voluntaria movimientos con lengua y labios. Boca en apertura permanente y babeo constante. Lengua recogida y bastante limitada en movimiento. Debilidad espástica. Ausencia de protusión. Respiración Oral. Debilidad en el soplo y de corta duración, afectando la coordinación neumofónica con baja capacidad respiratoria. Hipofonía y resonancia nasal. Movilidad de órganos fonoarticulatorios (labios, lengua y velo palatino) severamente comprometida tanto para el habla como para las actividades alimenticias. Succión débil con pitillo pero las bebidas ofrecidas en recipiente, simplemente las vierte en la boca. Alimentación de consistencia blanda básicamente. La mordida es débil y no hay movimiento masticatorio: ablanda los alimentos con la saliva y con cortos movimientos de lengua. Disfagia con riesgo frecuente de broncoaspiración. Sensación de ahogamiento. Tos débil. Desautomatización de la fase voluntaria de la deglución.

La paciente asistió a varias sesiones de terapia articulatoria impartida por fonoaudiología, encaminada a mejorar las condiciones alimentarias para disminuir los riesgos de la broncoaspiración y sensación de ahogamiento, mejorar su capacidad respiratoria y coordinación neumofónica, estimular la actividad de los músculos involucrados en el habla y alimentación, ejercitar puntos y modos articulatorios. Se ha logrado mejorar el control del cierre bucal, la intensidad y duración del soplo y de la fonación con prolongaciones vocálicas, la funcionalidad de la actividad motora para la alimentación disminuyendo la frecuencia de los ahogos; ha mejorado un poco el patrón de la deglución pero no se ha logrado automatizar, ha disminuido el babeo, sin mejoría de la anartria, ha logrado apretar y soltar dientes pero sin el movimiento asociado de apertura labial, algo de movilidad ascendente de lengua, puede mantener el cierre bucal completo (labios y dientes) mientras escribe lo que se le dicta o produce de manera espontánea (antes escribía con la boca abierta permitiendo la salida abundante de la saliva). Se han logrado algunos fonemas más claros $(/ \mathrm{a}-\mathrm{e}-\mathrm{m}-\mathrm{l})$. La paciente continúa comunicándose por escrito.

\section{Discusión}

Semiológicamente, la parálisis de pares craneales bajos por lesión supranuclear, que afecta a las fibras corticobulbares y corticopontinas, se conoce como parálisis supra o pseudobulbar. El síndrome de Foix-Chavany-Marie (FCM) o síndrome biopercular fue descrito como una diplejía labio-facio-faringo-laringo-gloso-masticatoria, con disociación automática del movimiento, sin alteración mental ni incontinencia esfinteriana ${ }^{1,2}$. Los pacientes que padecen este síndrome presentan alteración del habla (disartria o anartria), disfagia y no pueden abrir la boca, cerrar los párpados o protruir la lengua por voluntad propia, pero pueden sonreír, llorar y bostezar automáticamente.

El opérculo cerebral comprende una región que cubre la ínsula. Según Penfield y Rassmusen, se distinguen tres partes ${ }^{3}$ : el opérculo frontal, (áreas 44 y 45) extremidad caudal de la circunvolución frontal inferior; el opérculo parietal, base de la circunvolución precentral y poscentral que abarca parte del área suplementaria sensitiva y motora, $y$ el opérculo temporal, parte rostral de la circunvolución temporal superior (área 22) que mantiene conexiones con los opérculo frontal y parietal, la ínsula y el área 46. En el síndrome biopercular se interrumpen, en forma bilateral, las conexiones entre las áreas de la corteza motora y los núcleos de los pares craneales V, VII, IX, X y XII del tallo cerebral, lo que origina la diplejía oro-facial sin denervación ${ }^{3-5}$. La disociación automática voluntaria que caracteriza a este síndrome ha sido explicada por la diferenciación anatómica de las vías voluntarias y emocionales del control del movimiento de la musculatura facial.

Los músculos faciales y orales tienen dos controles centrales, un control voluntario que requiere de una corteza motora intacta y el control emocional que involucra estructuras profundas del sistema extrapiramidal, tálamo e hipotálamo. La base anatomofisiológica de la disociación automática-voluntaria, radica en que las proyecciones córtico-bulbares conducen los impulsos para la motilidad facial voluntaria mientras que los impulsos para los movimientos emocionales se transmiten al núcleo facial a través del sistema extrapiramidal y diencefálico ${ }^{3-6}$.

La etiología más frecuente en la edad adulta es la isquémica, a nivel opercular bilateral, pero también se ha descrito en pacientes con lesiones 
subcorticales bilaterales ${ }^{7}$ infecciosas ${ }^{6,8-10}$ con lesiones tumorales ${ }^{11}$, epilépticas ${ }^{12,13}$ o en trastornos de la migración neuronal ${ }^{14-16}$. Se han descrito formas del síndrome con parálisis facial, lingual y braquial unilateral por lesiones únicas contralaterales ${ }^{11}$. Son pocos los casos informados de síndrome biopercular con lesiones unilaterales ${ }^{7,17}$.

La mayoría de los pacientes informados en la literatura con síndrome biopercular han sido documentados con TC de cráneo. Son pocos los pacientes con este síndrome documentados con resonancia magnética de encéfalo ${ }^{7}$ en quienes se comprobó lesión unilateral exclusivamente. Pero la resonancia magnética también ha fallado para mostrar el compromiso bilateral demostrado por SPECT $^{18}$. En esta paciente, no sólo la resonancia magnética mostró la lesión unilateral derecha sino que SPECT mostró hipoperfusión en un solo lado (el derecho). A pesar de esto, no es posible, con los métodos diagnósticos usados, descartar definitivamente una lesión ya sea isquémica o de otro tipo en el lado contrario. En algunos estudios, la sensibilidad y especificidad de la técnica de SPECT para enfermedades neurodegenerativas con daños cerebrales difusos está alrededor de $80 \%$, siendo estas patologías conocidas por la disminución del flujo sanguíneo regional ${ }^{19}$. Sin embargo, varios estudios han demostrado la superioridad del SPECT frente a la RM para detectar las lesiones estructurales y funcionales ${ }^{18,20}$. En nuestro medio no contamos con tomografía por emisión de positrones (PET) el cual podría informarnos si en esta paciente hay hipometabolismo en el opérculo izquierdo a pesar de no observar lesiones en la resonancia magnética de encéfalo o alteración en la perfusión con la SPECT.

Con las herramientas diagnósticas usadas en esta paciente, se sugiere entonces la posibilidad de existencia del síndrome biopercular con lesión unilateral. La razón por la cual una lesión unilateral puede producir una parálisis bilateral de la musculatura facial-labial-masticatoria-faríngea y laríngea no ha sido explicado porque las aferencias a los núcleos motores de los pares craneales $\mathrm{V}$, VII, IX, X, XI, XII son generalmente bilaterales y cuando hay una lesión opercular, la corteza contralateral suple la función de la corteza dañada.

Una hipótesis sería la planteada por MoragasGarrido y $\mathrm{col}^{7}$, al establecer la posibilidad de la existencia de una variante anatómica en ciertos individuos de manera que la representación del fascículo geniculado fuese predominantemente unilateral y que la lesión de la corteza más involucrada en la inervación motora de los núcleos de los pares craneanos produjese manifestaciones bilaterales. A la luz de los conceptos anatomofisiológicos actuales es inexplicable la existencia de un síndrome biopercular por lesión unilateral. Futuras investigaciones con nuevas herramientas de trabajo como la resonancia magnética funcional o la tractografia nos podrían ayudar a comprender la razón de esta particular, escasa e interesante forma de presentación del síndrome descrito casi un siglo atrás.

Agradecimientos: Deseamos agradecer a COLCIENCIAS y la Universidad de Antioquia por la financiación del proyecto: 1115-343-19127 y al CODI por la financiación del programa de SOSTENIBILIDAD 2009-2010 del grupo de Neurociencias de Antioquia.

\section{Referencias}

1. Foix C, Chavany JA, Marie J. Diplégie facio-linguomasticatrice d'origine cortico-sous-corticale sans paralysie des membres. Rev Neurol (París) 1926; 33: 214-9.

2. De Smet Y. The anterior operculum syndrome. J Neurol 1994; 241: 570-1.

3. Bruyn GW, Gathier JC. The operculum syndrome. In Vinken PJ, Bruyn GW, ed. Handbook of Clinical Neurology. Localization in clinical neurology. Vol. 2. Amsterdam: North Holland Publishing; 1969. p. 776-87.

4. Mao CC, Coull BM, Golper LA, Rau MT. Anterior operculum syndrome. Neurology 1989; 39: 1169-72.

5. Bakar M, Kirshner HS, Niaz F. The opercularsubopercular syndrome: four cases with review of the literature. Behav Neurol 1998; 11: 97-103.

6. Moodley M, Bamber S. The Operculum syndrome: an unusual complication of tuberculosis meningitis. Dev Med Child Neurol 1990; 32: 919-22.

7. Moragas-Garrido M, Cardona-Portela P, Martínez-Yélamos S, Rubio-Borrego F. Heterogeneidad topográfica del síndrome de Foix-Chavany-Marie. Neurología 2007; 22: 333-6.

8. Cosnett JE, Moodley M, Bill PL, Bullock R. Operculum syndrome from brain abscess in a lefthander. J Neurol Neurosurg Psychiatry 1988; 51: 307-8.

9. Jadraque-Rodríguez R, Porta-Etessam J, Llana-Martín I, Martínez-Salio A, Torres-Mohedas J, Simón-de-lasHeras R, et al. Síndrome opercular secundario a encefalitis aguda en la infancia. Rev Neurol 2001; 33: 979. 
10. McGrath NM, Anderson NE, Hope JKA, Croxon MC, Powel KF. Anterior opercular syndrome caused by herpes simplex encephalitis. Neurology 1997; 49: 4947.

11. Alajouanine TH, Boudin G, Pertuiset B, Pépin B. Le syndrome unilatéral de l'opercule rolandique avec atteinte contralatérale du territoire des V, VII, IX, X, XI et XII nerfs craniens. Rev Neurol (París) 1959; 101: 168-71.

12. Colamaria V, Sgro V, Caraballo R, Simeone M, Zullini E, Fontana E, et al. Status epilepticus in benign rolandic epilepsy manifesting as anterior operculum syndrome. Epilepsia 1991; 32: 329-34.

13. Prats JM, Garaizar C, García Nieto ML, Madoz P. El síndrome opercular epiléptico: una forma peculiar de epilepsia parcial benigna de la infancia con paroxismos rolándicos. Rev Neurol 1999; 29: 375-80.

14. Millán PA, Montes MI, Uribe CS, Cabrera D, Arboleda A. Síndrome biopercular: presentación de dos casos y revisión de la literatura Biomédica. 2008 Jun; 28(2): 183-90.
15. Shevell MI, Carmant L, Meagher-Villemure K. Developmental bilateral perisylvian dysplasia. Pediatr Neurol 1992; 8: 299-302.

16. Márquez-Belandria G, Rondón-Hernández F, PascualCastroviejo I. Síndrome opercular bilateral congénito: presentación de tres casos. Rev Neurol 2003; 36: 938 40.

17. Starkstein SE, Berthier M, Leiguarda R. Bilateral opercular syndrome and crossed aphemia due to a right insular lesion: A clinicopathological study. Brain and Language 1988, 34: 253-61.

18. Kutluay E, Colako lu Z, Dirlik A, Kumral K. Brain SPECT in anterior opercular syndrome due to a unilateral lesion. J Neurol 1996; 243: 427-9

19. Petrella JR, Coleman RE, Doraiswamy PM. Neuroimaging and Early Diagnosis of Alzheimer Disease: A Look to the Future. Radiology. 2003; 226: 315-36.

20. Lampl Y, Steinmetz A, Gilad R, Eshel Y, Chamovitz D, Sarova-Pinhas I. 